\title{
Analysis of the influences of supplementing qi and nourishing yin formula on HR, ASP, ADP, LVSP, LVEDP and ET-1 of heart failure rats.
}

\author{
Luan Yingni* \\ Traditional Chinese Medicine Department, Shandong University Hospital, PR China
}

\begin{abstract}
Objective: To explore influences of supplementing qi and nourishing yin formula on death rate, cardiac function, haemodynamics and ET-1 of heart failure rats.

Methods: This study used ADR to establish heart failure model, they were divided into model control group, Shexiang Baoxin pill positive control group, high dose, medium dose and low dose experimental group of supplementing qi and nourishing yin formula, blank healthy control group. There were 12 rats in each group, half male and half female. They were given intragastric administration twice one day for 6 w continuously. Then we observed death conditions of rats, measured cardiac function of rats, including HR, ASP, ADP, LVSP, LVEDP and ET-1 level in serum.

Results: After administration of the last time, average weight and $H R$ of rats in different doses of supplementing qi and nourishing yin formula lower than the healthy control group, but LVEDP higher than the healthy control group, $\mathbf{P}<0.05$. ASP, ADP, LVSP, ET-1 level in serum of rats in high dose group were in accordance with healthy control group, P $>0.05$; but average weight, HR, ASP, LVSP, LVEDP and ET-1 level in serum all higher than model control group and positive control animal, $\mathbf{P}<0.05$. Average weight, various parameter of haemodynamics and ET-1 level in serum in medium and low dose group were in accordance with positive control group, $\mathbf{P}>\mathbf{0 . 0 5}$; but it higher than model control group, $\mathbf{P}<\mathbf{0 . 0 5}$.

Conclusion: Supplementing qi and nourishing yin formula can enhance myocardial function of $\mathrm{HF}$ rats, improve abnormal haemodynamics, lower ET level in serum, which has good clinical application prospect.
\end{abstract}

Keywords: Supplementing qi, Nourishing yin formula, HF, Rats experiment, Haemodynamics, ET 1.

Accepted on November 2, 2017

\section{Introduction}

Heart Failure (HF), TCM is also calls heart impediment, cardiac edema etc., it takes qi-deficiency and yang-deficiency as pathogenesis. The main symptoms in the middle and later stage have blood stasis, water retention, phlegm-dampness, weakness of the body and exogenous evil, which harm yang-qi in various viscera of heart, lung, spleen and kidney $[1,2]$. For HF treatment, TCM diagnosis in early stage is relative difficult. With the progress of conditions, it will have clinical indications of palpitation, fatigue, dyspnea etc. TCM suggests dialectical classify treatment. Whatever types, qi deficiency and blood stasis are the basis of HF onset. Therefore, supplementing qi, nourishing yin and activating blood method throughout the whole process $[3,4]$. Supplementing qi and nourishing yin formula in this study has better clinical effects on improving cardiac muscle function, relieving HF symptom. This study establishes rats HF model for exploring its function mechanism, combining with Western medicine furtherly, observing influences of supplementing qi and nourishing yin formula on death rate, cardiac function, haemodynamics and ET-1.

\section{Instruments and Materials}

\section{Subject animal}

Ethical approval was given by the medical ethics committee of Shandong University Hospital with the following reference number: 2016012. This selected 72 clean grade Wister rats of 160 to $200 \mathrm{~g}$, which provided by Beijing vital River animal center, fed in SPF animal room in this unit. The temperature in room was $24 \pm 5^{\circ} \mathrm{C}$. They were given day and night alternate lighting, drink and eat freely.

\section{Subject drug and main reagent}

First, ADR bought from Pfizer pharmaceuticals limited in Haizheng. Specification: $10 \mathrm{mg}$ per bottle. Approval number: SFDA number H33021980; second, shexiang baoxin pill bought from Hehuang pharmaceutical limited company in Shanghai, specification: $22.5 \mathrm{mg}$ per pill, specification: SFDA number Z31020068; third, supplementing qi and nourishing yin formula, including $15 \mathrm{~g}$ Radix liquiritiae, $15 \mathrm{~g}$ Astragalus, 15 g Radix codonopsis, 15 g dried Radix rehmanniae, $15 \mathrm{~g}$ 
Poria cocos, 10 g cassia twig, 10 g Radix paeoniae, 10 g Radix curcumae, $10 \mathrm{~g}$ fresh ginger and $5 \mathrm{~g}$ Fructus Ziziphi Jujubae; which was prepared by pharmacy department in our hospital according to technological process strictly, decocted by water, then concentrated to $2 \mathrm{~g} / \mathrm{ml}$ of crude drug. One dose one day. Twice per day; fourth, NS bought from Anhui double-crane pharm limited company. Specification: $500 \mathrm{ml}$. Approval number: SFDA number H20054037; fifth, ET-1 ELISA detection kits bought from Shanghai ExcellBio technology limited company.

\section{Main instruments}

First, XY-LD bought from magnetic flow meter from Jiangsu Xiyi instrument limited company; second, Ponemah multichannel physiological record instrument brought from Beijing Baianji technology limited company; third, Philips M3536A cardiac defibrillator-monitor bought from Shanghai Jieweifo medical devices limited company; fourth, small animal ventilator from Shanghai Yuyan scientific instrument limited company.

\section{Experimental methods}

Modeling methods: 60 rats were given $2 \mathrm{mg} / \mathrm{kg}$ ADR intraperitoneal injection once a week for 8 times in total. The accumulated dose reached to $16 \mathrm{mg} / \mathrm{kg}$, then rats had typical HF clinical symptom changes and pathological changes, including energielos, slow response, hair removal, inappetence, edema etc. For other 12 rats, given $1 \mathrm{ml} \mathrm{NS}$ intragastric injection once a week for 8 times in total.

Group: 72 subject rats were given weight measurement, they were divided into the blank control group, ADR model control group, Shexiang baoxin pill control group, supplementing qi and nourishing yin formula group (high, medium and low dose group) according to weight.

Administration method: Rats in the blank control group and ADR group were given intrasgastric NS twice one day about $10 \mathrm{ml} / \mathrm{kg}$. Rats in positive control group were given intragastric administration twice one day according to $24 \mathrm{mg} / \mathrm{kg} / \mathrm{d}$. supplementing qi and nourishing yin formula group (high, medium and low dose group) were four, two and one times than human equivalent dose. The volume of intragastric administration in rats was $1 \mathrm{ml} / 100 \mathrm{~g}$ twice per day for $6 \mathrm{w}$ continuously.

Detection indexes: After the last time intragastric administration, this study measured cardiac function of rats, including HR, ASP, ADP, LVSP and LVEDP, at the same time, collected blood in neck artery of rats, located in centrifuge tube with aprotinin and EDTA in precool environment, detected according to guide of ET-1 ELISA detection kits. Then this study monitored emotion state, water-depriving and fasting conditions, weight conditions, defecation stool quantity and death conditions etc.

\section{Statistical management}

This data adopted SPSS 19.0 software to do statistical analysis; homogeneity of variance used single factor ANOVA. Comparison between groups used LSD test. Heterogeneity of variance used rank-sum test; $\mathrm{P}<0.05$, there were statistical differences.

\section{Results}

\section{Weight changes of rats and death conditions}

After administration, there were no dead animal in the blank control group, model control group, positive control group, high dose, medium and low dose group of subject drug had 6 , 5, 2, 4 and 5 death animals respectively. After building model, the average weight of HF rats was $353.61 \pm 64.82 \mathrm{~g}$, compared with the blank control group, there were statistical differences, $\mathrm{P}<0.05$. After $6 \mathrm{w}$ of administration, animal in the blank control group grew normally. Weight of ADR model rats in each group lower than model control group, there were statistical differences, $\mathrm{P}<0.05$; average weight in positive control group and various subject group higher than model control group, there were statistical differences, $\mathrm{P}<0.05$; in addition, high dose group of supplementing qi and nourishing yin formula higher than positive control group, there were statistical differences, $\mathrm{P}<0.05$, Table 1 .

Table 1. Weight changes and death conditions of rats in each group (average value \pm standard deviation). Note: ${ }^{a}$ compared with the blank control group, $P<0.05 ;{ }^{b}$ compared with model control group, $P<0.05$; ${ }^{c}$ compared with positive control group, $P<0.05$.

\begin{tabular}{|c|c|c|c|c|c|c|c|}
\hline Group & $\begin{array}{l}\text { Animal } \\
\text { number } \\
\text { (number) }\end{array}$ & $\begin{array}{l}\text { Death } \\
\text { number } \\
\text { (number }\end{array}$ & $\begin{array}{l}\text { Weight } \\
\text { before } \\
\text { administrati } \\
\text { on }(\mathrm{g})\end{array}$ & & $\begin{array}{l}\text { Weight afte } \\
\text { Administratio } \\
\text { (g) }\end{array}$ & & $\begin{array}{l}\text { Change } \\
\text { rate of } \\
\text { weight } \\
(\%)\end{array}$ \\
\hline $\begin{array}{l}\text { Blank } \\
\text { control }\end{array}$ & 12 & 0 & $\begin{array}{l}407.49 \\
84.71\end{array}$ & \pm & $\begin{array}{l}518.92 \\
127.31\end{array}$ & \pm & +27.35 \\
\hline $\begin{array}{l}\text { Model } \\
\text { control }\end{array}$ & 12 & 6 & $\begin{array}{l}352.15 \\
59.62^{a}\end{array}$ & \pm & $389.56 \pm 99.82$ & $2^{a}$ & +10.62 \\
\hline $\begin{array}{l}\text { Positive } \\
\text { control }\end{array}$ & 12 & 5 & $\begin{array}{l}357.20 \\
61.53^{a}\end{array}$ & \pm & $\begin{array}{l}425.47 \\
123.56^{\mathrm{ab}}\end{array}$ & \pm & +19.11 \\
\hline High dose & 12 & 2 & $\begin{array}{l}350.59 \\
53.66^{a}\end{array}$ & \pm & $\begin{array}{l}474.89 \\
115.04^{\mathrm{abc}}\end{array}$ & \pm & +35.45 \\
\hline $\begin{array}{l}\text { Medium } \\
\text { dose }\end{array}$ & 12 & 4 & $\begin{array}{l}348.46 \\
62.38^{a}\end{array}$ & \pm & $\begin{array}{l}448.19 \\
134.27^{\mathrm{ab}}\end{array}$ & \pm & +28.62 \\
\hline Low dose & 12 & 5 & $\begin{array}{l}354.15 \\
73.18^{a}\end{array}$ & \pm & $\begin{array}{l}433.55 \\
109.59^{\mathrm{ab}}\end{array}$ & \pm & +22.42 \\
\hline
\end{tabular}

\section{Parameter comparison of haemodynamics of animal in various groups}

According to detection results, compared with haemodynamics of rats in normal control group, HR, ASP and LVSP in ADR model control group, positive control group, medium dose subject group and low subject group decreased, LVEDP increased, there were statistical differences, $\mathrm{P}<0.05$; HR in high dose subject group decreased, LVEDP increased, there 
were statistical differences, $\mathrm{P}<0.05$; other various parameter compared with blank control group, there were no statistical differences, $\mathrm{P}>0.05$; compared with rats in model control group, HR, ASP, ADP and LVSP in positive group, high, medium and low dose subject group increased, LVEDP decreased, there were statistical differences, $\mathrm{P}<0.05$; compared with rats in positive control group, HR, ASP and LVSP increased, LVEDP decreased, there were statistical differences, $\mathrm{P}<0.05$; various haemodynamics indexes of rats in medium, low subject group were in accordance with positive control group, there were no statistical differences, $\mathrm{P}>0.05$, Table 2 .

Table 2. Parameter comparison of haemodynamics in rats in each group (average value \pm standard deviation). Note: ${ }^{a}$ compared with the blank control group, $P<0.05 ;{ }^{b}$ compared with model control group, $P<0.05$; ${ }^{c}$ compared with positive control group, $P<0.05$.

\begin{tabular}{|c|c|c|c|c|c|c|c|c|c|}
\hline Group & $\begin{array}{l}\text { HR (tim } \\
\text { min) }\end{array}$ & & $\begin{array}{l}\text { ASP } \\
(\mathrm{mmHg})\end{array}$ & & $\begin{array}{l}\text { ADP } \\
(\mathrm{mmHg})\end{array}$ & & $\begin{array}{l}\text { LVSP } \\
(\mathrm{mmHg})\end{array}$ & & $\begin{array}{l}\text { LVEDP } \\
(\mathrm{mmHg})\end{array}$ \\
\hline \multirow[t]{2}{*}{ Blank control } & 135 & \multirow[t]{2}{*}{ \pm} & 130.77 & \multirow[t]{2}{*}{ \pm} & 83.51 & \multirow[t]{2}{*}{ \pm} & 257.84 & \multirow[t]{2}{*}{ \pm} & $4.19 \pm 0.97$ \\
\hline & 10.49 & & 10.54 & & 10.99 & & 34.73 & & \\
\hline \multirow[t]{2}{*}{ Model control } & & \pm & 92.39 & \pm & 63.26 & \pm & 196.74 & \pm & 14.83 \\
\hline & & & & & & & & & \\
\hline \multirow{2}{*}{$\begin{array}{l}\text { Positive } \\
\text { control }\end{array}$} & & \pm & 110.12 & \pm & 81.61 & \pm & 225.54 & \pm & 9.00 \\
\hline & $30.67^{\mathrm{ab}}$ & & $19.86^{a b}$ & & $12.74^{\mathrm{b}}$ & & $29.68^{a b}$ & & $1.36^{\mathrm{ab}}$ \\
\hline \multirow[t]{2}{*}{ High dose } & 102 & \pm & 122.25 & \pm & 81.75 & \pm & 239.15 & \pm & 6.27 \\
\hline & $29.86^{a b c}$ & & $19.41^{b c}$ & & $16.23^{b}$ & & $30.77^{b c}$ & & $1.32^{\mathrm{abc}}$ \\
\hline \multirow[t]{2}{*}{ Medium dose } & 84 & \pm & 113.36 & \pm & 78.49 & \pm & 227.28 & \pm & 8.94 \\
\hline & $28.32^{a b}$ & & $22.74^{\mathrm{ab}}$ & & $13.86^{b}$ & & $34.26^{a b}$ & & $1.79^{a b}$ \\
\hline \multirow[t]{2}{*}{ Low dose } & 71 & \pm & 110.83 & \pm & 79.23 & \pm & 216.62 & \pm & 9.38 \\
\hline & $33.65^{a b}$ & & $25.02^{a b}$ & & $17.51^{\mathrm{b}}$ & & $29.71^{a b}$ & & $2.54^{\mathrm{ab}}$ \\
\hline
\end{tabular}

\section{Comparison of ET-1 level in serum of animal in each group}

According to detection results, compared with rats in normal control group, ET-1 level in ADR model control group, positive control group, medium and low subjects increased, there were statistical differences, $\mathrm{P}<0.05$; high dose subject group compared with blank control group, there were no statistical differences, $\mathrm{P}>0.05$; compared with model control group, ET-1 level in model control group, high, medium and low dose subject decreased, there were statistical differences, $\mathrm{P}<0.05$; compared with positive control group, serum ET-1 level in high dose subject group decreased, there were statistical differences, $\mathrm{P}<0.05$; but various haemodynamics indexes in low dose subject group were in accordance with positive group, there were no statistical differences, $\mathrm{P}>0.05$, Table 3 .

Table 3. Comparison of serum ET-1 level of rats in various groups (average value \pm standard deviation). Note: ${ }^{\text {a }}$ compared with the blank control group, $P<0.05 ;{ }^{b}$ compared with model control group, $P<0.05$; ${ }^{c}$ compared with positive control group, $P<0.05$.

\begin{tabular}{lllllll}
\hline $\begin{array}{l}\text { Detectio } \\
\mathbf{n}\end{array}$ & $\begin{array}{l}\text { Blank } \\
\text { control }\end{array}$ & $\begin{array}{l}\text { Model } \\
\text { control }\end{array}$ & $\begin{array}{l}\text { Positive } \\
\text { control }\end{array}$ & High dose & $\begin{array}{l}\text { Medium } \\
\text { dose }\end{array}$ & $\begin{array}{l}\text { Low } \\
\text { dose }\end{array}$ \\
\hline
\end{tabular}

\begin{tabular}{|c|c|c|c|c|c|c|c|c|c|}
\hline $\begin{array}{l}\text { ET-1 } \\
\text { (ng/l) }\end{array}$ & $\begin{array}{l}32.57 \\
14.17\end{array}$ & & $\begin{array}{l}88.65 \\
36.23^{a}\end{array}$ & \pm & $\begin{array}{l}51.76 \\
19.38^{\mathrm{ab}}\end{array}$ & $\begin{array}{l}40.41 \\
15.63^{b c}\end{array}$ & \pm & $\begin{array}{l}50.39 \\
17.26^{\mathrm{ab}}\end{array} \pm$ & $\begin{array}{l}55.54 \\
19.82^{\mathrm{ab}}\end{array} \pm$ \\
\hline
\end{tabular}

\section{Discussion}

HF refers to heart-qi deficiency, yang-qi deficiency, which caused by heart malnutrition, relating to heart, spleen, lung and kidney, it causes unsmooth of blood, stasis of phlegmdampness and water-dampness failing to transform etc. [5]. In ancient times, in Neijing, there are records that heart water, will cause heavy body and shortness of qi, unable to lay down, tired and vulval swelling, which are in accordance with HF theory in modern Western medicine [6,7]. In song dynasty, general collection for holy relief of heart door record that HF will cause amnesia, deficiency will cause dull pain in chest, abdomen, subcostal part, lumbar part and back, palpitation, ecstasy, short of color [8]. From that, we can see that ancient TCM theory already mentioned HF concept, and think that pathogenesis have heart-qi and yang deficiency, which cause blood stasis, phlegm dampness and edema. TCM thinks that heart controls blood and meridian [9]. If there are heart-qi deficiency, heart-yang deficiency, which will influence blood circulation of whole body, degeneration of abdomen and viscera, dysfunction of various physiological activity.

In recent years, our country combine TCM theory with Western medicine gradually, thinks that qi-deficiency has relations with dysfunction of heart in different degrees and abnormal haemodynamics [10], which include abnormal systolic function, diatolic function and pump blood, decreased heart stroke volume, relative deficiency of tissue perfusion, it is one of important pathogenesis basis of HF [11]. Therefore, in theory, supplementing qi and nourishing yin formula not only can improve qi-deficiency, also can improve cardiac function and abnormal haemodynamics [12]. This study also shows that comparing with haemodynamics parameter of rats in normal control group, HR, ASP and LVSP in ADR model control group, positive control group, medium dose subject group and low subject group decrease, LVEDP increases, $\mathrm{P}<0.05$; HR in high dose subject group decreases, LVEDP increases, $\mathrm{P}<0.05$; other various parameter comparing with blank control group, there are no statistical differences, $\mathrm{P}>0.05$; comparing with rats in model control group, HR, ASP, ADP and LVSP in positive group, high, medium and low dose subject group increased, LVEDP decrease, $\mathrm{P}<0.05$; comparing with rats in positive control group, HR, ASP and LVSP increase, LVEDP decrease, $\mathrm{P}<0.05$; but various haemodynamics indexes in low dose subject group are in accordance with positive control group, there are no statistical differences, $\mathrm{P}>0.05$. It shows supplementing qi and nourishing yin formula relieve HF symptoms of heart-qi deficiency by improving systolic and diastolic function of left ventricle for increasing heart export volume.

Different from Western medicine theory, TCM in treating HF mainly emphasize general regulation of body, which not only pay attention to lesion of heart, and much to relations between heart and other viscera $[13,14]$. Especially in recent years, it more emphasize dynamic thinking, considering different stages 
of disease progress, we should predict progress direction of HF and regularity promptly to adopt more proper treatment methods. Whatever condition of disease progress, treatment should base on qi-deficiency, heart-kidney yang deficiency, supplementing qi and nourishing yin formula goes through all the time. Supplementing qi and nourishing yin formula is a kind of formula by the experience of treating HF by clinical doctors in our hospital, including Radix liquiritiae, Radix codonopsis, Poria cocos, Raidix paeoniae alba, ginger and jujube, which have function of tonifying yuan-qi, supplementing spleen and lung, soothing blood and meridian, stool and urine.

In addition, there are studies comparing with modern pathological study demonstrate that ET has relations with HF $[15,16]$. ET-1 not only has strong systolic function for blood, also promote growth of smooth muscle and ANP secretion, which is one of typical inflammatory influencing factors $[17,18]$. This study also analyses the influences of supplementing qi and nourishing yin formula on ET-1 simply. Results show that comparing serum ET-1 level in high dose subject group with blank control group, there are no statistical differences, $\mathrm{P}>0.05$; but it lower than model control group and positive control group, $\mathrm{P}<0.05$; but various haemodynamics indexes in low and medium dose subject group are in accordance with positive control group, there were no statistical differences, $\mathrm{P}>0.05$. It shows that the treatment effects of supplementing qi and nourishing yin formula on Shexiang Baoxin pill.

In conclusion, through experiment in vivo of rats, this study explores influences of supplementing qi and nourishing yin formula on cardiac function of HF rats model, haemodynamics and ET-1, which shows that supplementing qi and nourishing yin formula can enhance cardiac function of HF patients, relieve abnormal haemodynamics, lower serum ET-1 level to provide theory support for HF treatment in clinic.

\section{References}

1. Zhang N, Chen SJ, Dong ZZ. Clinical evaluation of HF on TCM diagnosis methods. Chinese J Integr Med Cardio/ Cerebrovasc Dis 2017; 15: 590-592.

2. Zhao C, Wang YY. Research progress on mechanism of benefiting qi for activating blood circulation chinese medicine in the treatment of heart failure. Chinese Med Mod Dist Edu China 2017; 15: 139-140.

3. Wang XL. Effects study on TCM with Western medicine in treating HF. Practical Clinic J Integr Trad Chinese West Med 2017; 17: 49-50.

4. Liu MX, Hu B. The influences of supplementing qi and nourishing yin formula on haemodynamics, cardiac function indexes and life quality of acute HF patients with rheumatic heart diseases. J Emerg Trad Chinese Med 2015; 24: 2183-2185.
5. Li L, DU HY, Liu LZ. Observation of the curative effect of Yiqi Yangyin Huoxue therapy on chronic heart failure. Mod J Integr Trad Chinese West Med 2016; 25: 152-154.

6. Liu LL, Miao Y, Wang J. Observation of the curative effect of Yiqi Yangyin Huoxue therapy on chronic heart failure. Mod J Integr Trad Chinese West Med 2014; 12: 153-155.

7. Huang CY, Li YZ. Discussion and treatment of HF. Jilin J Trad Chinese Med 2011; 31: 19-25.

8. Huang J. Basic theory of chronic HF treatment: past; present; future. Tianjing Med 2016; 44: 929-931.

9. Zhang Q, Zhang Y, Gong LH. Clinical study on three stages of TCM in treating chronic HF. J Liaoning Univ Trad Chinese Med 2016; 18: 178-180.

10. Haneer DMT, Ma HT, Wang HY. Study progress on physiological and onset progress of chronic HF. Chinese J Integr Med Cardio/Cerebrovasc Dis 2016; 14: 1349-1352.

11. Salamanca BP, Aramburu BO, Formiga F. Heart failure: does it matter which speciality treats it? Rev Esp Geriatr Gerontol 2017.

12. Sun W, He Y, Yu J, Lin Y, Wang Y. Effect of yiqiyangyin recipe on heparanase and nephrin in rats with adriamycininduced nephropathy. J Tradit Chin Med 2013; 33: 334-342.

13. Chen H, Li C, Shi TQ. Influence of Qishen Yiqi Gutta Pills on RAAS in rats with heart failure. J Beijing Univ Trad Chinese Med 2014; 37: 538-542.

14. Yuan W, Jin M, Pan L. Influence of Qishenyiqi Gutta Pills on expression of ICAM-1 in rats with diabetic retinopathy. J Beijing Univ Trad Chinese Med 2010; 33: 262-265.

15. Xiong B, Nie D, Cao Y. Clinical and hemodynamic effects of endothelin receptor antagonists in patients with heart failure. Int Heart J 2017; 58: 400-408.

16. Greenberg BH. Endothelin and endothelin receptor antagonists in heart failure. Congest Heart Fail 2010; 8: 257-261.

17. Jankowich MD, Wu WC, Choudhary G. Association of elevated plasma endothelin-1 levels with pulmonary hypertension, mortality, and heart failure in African American individuals: the Jackson heart study. JAMA Cardiol 2016; 1: 461-469.

18. Rodrigues MC, Cooke RF, Marques RS. Effects of vaccination against respiratory pathogens on feed intake, metabolic, and inflammatory responses in beef heifers. J Anim Sci 2015; 93: 4443.

\section{*Correspondence to}

Luan Yingni

Traditional Chinese Medicine Department

Shandong University Hospital

PR China 